\title{
Routine-Based View of Organizational Learning and Mechanisms of Myopia
}

Hidenori SATO ${ }^{a}$

\begin{abstract}
Levinthal and March (1993) are popularly supposed to have developed a concept "myopia of learning" to reveal negative aspects of organizational learning. However, myopia of learning is not a problem in itself. The myopia of learning means that organizations give precedence to exploitation over exploration. From a routine-based view of organizational learning, myopia of learning only becomes a problem when the mechanisms promoting exploitation take precedence over the mechanisms promoting exploration at all levels of the organization.
\end{abstract}

Keywords: organizational learning, myopia of learning, routine

\section{Introduction}

Some studies cite Levinthal and March (1993) as identifying the negative aspects of learning because of the term 'myopia'. However, Levinthal and March essentially advocated a realistic theory on

a) Faculty of Economics, Nagasaki University, hidesato@nagasaki-u.ac.jp

A part of this paper was originally published as Sato (2008) in Japanese. 
organizational learning, which states that there are limitations to learning.

Myopia only becomes problematic in certain conditions; myopic behavior in itself is not problematic for an organization.

It is more important to be aware that learning mechanisms themselves can cause myopia, and consider how to make the best use of organizational learning, which is essentially limited.

\section{The Myopia of Learning}

Learning is not all-powerful. Even the mechanisms that promote learning can contain limiting factors and cause myopia. Levinthal and March advocated three types of myopia of learning: (a) temporal myopia, (b) spatial myopia, and (c) failure myopia.

Temporal myopia means overlooking distant times. Learning tends to sacrifice the long run for the short run (short term > long term). Although the learner may acquire unique capabilities, the skills learned may become an obstacle in the long term.

Spatial myopia means overlooking distant places and the larger picture. Often the best strategies for individual parts of the system are not the best strategies for the entire system. Consequently, promoting learning in the individual parts of the system can sometimes cause problems in the overall system (parts > whole).

Failure myopia means overlooking the failures in the organization in contrast to successes. If the organization continues to succeed through learning, this also builds up capability and confidence. A successful person has power within an organization and a definite influence on organizational decisions. Consequently, the organization tends to become biased toward emphasizing past successes over past failures, and tends to ignore risks (success > failure).

The limitations on learning advocated by Levinthal and March are 
based on the idea that these myopias of learning are caused by the mechanisms used to promote learning.

\section{Two Mechanisms of Learning}

\subsection{Simplification}

An organization must interpret its experiences to learn. To do this, the organization must simplify complex environments to be recognized. There are two methods to achieve this, given below.

\section{(i) construction of buffers}

The environment can be simplified by departmentalization and sequential attention, rather than aiming for multiple goals at once.

\section{(ii) enactment}

The effectiveness of the construction of buffers depends on the divisibility of the problems. It cannot function when the problems cannot be divided. However, the divisibility of the problems depends on the cognitive framework of the organization toward its environment. Different organizations draw different meanings from the same situation. Consequently, the divisibility is determined not as an external technical problem, but as an organizational problem.

\subsection{Specialization}

If an entire system such as an organization attempts to adapt to the environment all at once, the interaction between the parts in the system becomes complicated and unstable. Assuming that each of the parts adapts to their environments to promote learning, the success in adapting one part within the system has two effects: relieving the pressure for adaptation in another part, and developing a greater adaptation capacity than other parts. These combine to 
produce learning that is specialized in its parts.

\section{Mechanisms of Myopia}

As mentioned above, an organization can use simplification and specialization to promote learning. However, Levinthal and March assert that this in itself can produce myopia.

A simplification of the environment to enact is the effectiveness in promoting learning. However, adapting to a simplified environment, if the enactment from past environments is no longer effective, then the previous build up of capabilities is likewise no longer effective. By specializing, organizations can build up capabilities and promote learning in certain areas. The organization can also take learning in a particular field to a higher level, because using existing skills yields greater short-term returns than developing new capabilities, and learning in one part can be substituted for learning in another part. However, this also means a curtailment of learning in areas outside of the specialized field. Consequently, if the environment changes in the long term, specialization and building up capabilities in a certain field is counterproductive.

It is also possible that promoting learning in parts of the system through simplification or specialization can pose a threat to the entire system. Promoting learning in a particular part curtails learning in other parts. Consequently, although parts may build up capabilities through learning, it is not necessarily total optimization. This is true at organization level and inter-organization level. At inter-organization level, some organizations may feel it is appropriate to specialize and not carry out research in new fields. However, if all organizations choose to do the same, nobody will research new fields and the entire system will have underinvested in research. Research involves not only producing new information but also improving the capability to absorb information from other companies. The greater 
the pool of information, including that of other companies, the greater the incentive for research. However, if no companies engage in new research, this creates a vicious cycle in which the pool of information does not grow and the incentive for investing in research decreases.

Failure myopia is also caused by promoting learning in a particular field. Increasing capabilities through learning reduces the chance of failure, instilling greater confidence in an organization and the individuals in it. In an organization, a successful person is promoted and gains greater privileges. As a result, there is a danger of becoming over-confident and ignoring risks.

\section{Exploitation and Exploration}

In order to understand the myopia of learning, it is necessary to understand the concepts of exploitation and exploration. This is because the mechanisms stated by Levinthal and March to cause myopia are more or less the same as "exploitation favored over exploration". Levinthal and March define exploitation as "the use and development of things already known" and exploration as "the pursuit of new knowledge and of things that might come to be known" (Levinthal \& March, 1993, p. 105).

Levinthal and March (1993) call the excess of either exploitation or exploration as the traps of learning (Levinthal \& March, 1993, p. 105). Short-term positive feedback gives rise to further exploitation or exploration, and upsets the balance between the two.

Since exploration and exploitation are both able to upset the balance, Levinthal and March call mechanisms that cause excess exploration the failure trap (Levinthal \& March, 1993, p. 105), and mechanisms that cause excess exploitation the success trap (Levinthal \& March, p. 106).

The failure trap means falling into a vicious cycle of failure 
producing exploration, leading to further exploration once that fails. Typically, new ideas are more likely to fail. Even great ideas do not benefit much until some degree of experience can be built up. Consequently, when there is an optimistic bias and proactive approach toward exploration, there is a tendency to embark on fresh exploration, rather than gain experience based on existing ideas. This results in a repeated failure.

The success trap means short-term success gained by exploitation, which leads to further emphasis on exploitation. Exploration of new ideas has no short-term benefits and has a high degree of uncertainty. On the other hand, exploitation of existing knowledge and technologies brings short-term benefits, and so is favored. Further, knowledge and technologies are built upon through exploitation, and the opportunity cost of exploring new knowledge increases.

Levinthal and March believe that exploitation is far more likely to be in excess. According to them, although learning is gained through experience, exploitation brings feedback more clearly and quickly than exploration. Accordingly, there is a tendency of rapid learners to reduce the resources allocated to exploration. This results in excessive exploitation.

\section{Does the Myopia of Learning Matter?}

These are the myopia of learning and its causal mechanisms advocated by Levinthal and March. However, the failure trap is a mechanism in which exploration exceeds exploitation. This could also be called "myopic" in the sense that new exploration in the short term has priority to building up experience in the long term. In other words, Levinthal and March are only advocating one part of the consequences of myopic behavior. Why do they call it myopia when exploitation is in excess? This is the influence of March's view on organizational learning. 
March views organizational learning as an ecological transition of a routine within an organization (Levitt $\&$ March, 1988; March, 1991). ${ }^{1}$ According to Levitt and March (1988), there are three thoughts on which organizational learning is based: (1) routine-based thoughts; (2) history-dependent thoughts; and (3) target-oriented thoughts.

If we treat learning as routine based and ecological, we can view organizational learning as the variation and selection of new routines. Accordingly, March treats the relationship between exploration and exploitation much in the same way as that between variation and selection (March, 1991, p. 73). Combined with the fact that the relationship between exploration and exploitation can explain the mechanisms that cause myopia, March's logic indicates that from an ecological viewpoint, the myopia of learning is caused by the relationship between variation and selection. In other words, myopia is caused on the basis of March's view on organizational learning.

However, in a sense this is natural, the ecological way of thinking holds that adaptation takes place in a higher system due to selection at a lower level. In this case, the action taking place at the lower level does not need to be done with any thought for the system as a whole. In other words, the ecological adaptation process is usually "myopic".

March's view on organizational learning focuses on convergence rather than divergence, even in the ecological adaptation process. This is probably the reason why Levinthal and March treat myopia as being an excess of exploitation rather than exploration.

However, myopia in itself is not a problem. Since March's view on organizational learning tends to advocate an excess of exploitation, Levinthal and March (1993) also treat over-adaptation as a problem, and do not acknowledge under-adaptation. However, if we look at the respective phases, it is normal for both over-adaptation and under-adaptation to occur; this does not necessarily indicate

1 Although this is a similar, typical theory on organizational learning, it differs from other studies such as Argyris and Shön (1978). 
maladaptation.

In terms of levels of an organization, the conditions in which Levinthal and March's myopia presents a problem are more limited. According to their argument, division-level selection takes place within an entire company, while selection of technologies takes place within a division. However, these do not necessarily take place at the same time. Multiple technologies coexisting within a division even when division-level selection has taken place ensures diversity at enterprise level. In other words, myopia only becomes a problem when the mechanisms promoting exploitation take precedence over the mechanisms promoting exploration at all levels of the organization.

Levinthal and March (1993) point out that the logic in mechanisms for promoting learning can cause limitations. However, based on this logic, there are only limited situations in which myopia becomes a problem.

In other words, rather than clarifying the negative aspects of learning, Levinthal and March indicated a need to consider how to carry out learning, which has essentially myopic characteristics, in the 1990s when organizational learning was gaining more attention (Ando, 2002).

In terms of the responses of organizations to changing environment based on routine, ${ }^{2}$ Levinthal and March's argument has much in common with the concepts of organizational capability and dynamic capability. ${ }^{3}$ The implications for these arguments are still very significant today.

2 The view of organizations based on routine is influenced by Cyert and March (1963) and Nelson and Winter (1982).

3 Typical studies include Eisenhardt and Martin (2000), Teece, Pisano and Shuen (1997), Winter (2003), and Zollo and Winter (2002). Empirical research on organizational capability includes Henderson and Cockburn (1994). Studies focusing on Japanese companies include Fujimoto (1999), Kuwashima (2003), and Tomita (2009). 


\section{References}

Ando, F. (2002). The real relationship between organizational culture and organizational learning. Annals of Business Administrative Science, 1, 25-34.

Argyris, C., \& Shön, D. (1978). Organizational learning. Reading, MA: Addison-Wesley.

Cyert, R. M., \& March, J. G. (1963). A behavioral theory of the firm. Englewood Cliffs, NJ: Prentice-Hall.

Eisenhardt, K., M., \& Martin, J. A. (2000). Dynamic capabilities: What are they? Strategic Management Journal, 21(10-11), 1105-1121.

Fujimoto, T. (1999). The evolution of a manufacturing system at Toyota. New York, NY: Oxford University Press.

Henderson, R., \& Cockburn, I. (1994). Measuring competence: Exploring firm effects in pharmaceutical research. Strategic Management Journal, 15(S1), 63-84.

Kuwashima, K. (2003). Organizational capability and competitive advantage in pharmaceutical product development. Annals of Business Administrative Science, 2, 21-27.

Levinthal, D. A., \& March, J. G. (1993). The myopia of learning. Strategic Management Journal, 14(S2), 95-112.

Levitt, B., \& March, J. G. (1988). Organizational learning. Annual Review of Sociology, 14, 319-340.

March, J. G. (1991). Exploration and exploitation in organizational learning. Organization Science, 2(1), 71-87.

Nelson, R. R., \& Winter, S. G. (1982). An evolutionary theory of economic change. Cambridge, MA: Harvard University Press.

Sato, H. (2008). Machi no soshiki gakushukan to gakushu no kinshigan [March's view of organizational learning and the myopia of learning: Does the myopia matter?]. Akamon Management Review, 7(6), 409-418 (in Japanese).

Teece, D. J., Pisano, G., \& Shuen, A. (1997). Dynamic capabilities and strategic management. Strategic Management Journal, 18(7), 509-533.

Tomita, J. (2009). New product development and evaluating capabilities: The case of the material industry. Annals of Business Administrative 
Science, 8, 43-54.

Winter, S. G. (2003). Understanding dynamic capabilities. Strategic Management Journal, 24(10), 991-995.

Zollo, M., \& Winter, S. G. (2002). Deliberate learning and the evolution of dynamic capabilities. Organization Science, 13(3), 339-351.

Received February 2, 2012; accepted April 3, 2012 